\title{
Growing Skull Fracture [GSF], a Hydrodynamic Perspective: An Original Article
}

\author{
Allan J Drapkin, $M D, F A C S(R)^{1,2 *}$ iD \\ ${ }^{1}$ Department of Neurosurgery, Rutgers Biomedical and Health Sciences University, USA \\ `Jersey Shore University Medical Center, USA
}

\begin{abstract}
Growing skull fracture (GSF) is a well-known complication of pediatric cranial trauma but its physiopathology has been, so far, only assumed. When the basic laws of hydraulics are applied to the underlying GSF pathology, a clearer understanding of the process involved in it emerges and accounts for the frequent spontaneous arrest of the skull erosion. Moreover the process causing the commonly associated intra-axial pathology is also better defined.
\end{abstract}

Keywords

Head trauma, Skull fracture-skull erosion, Leptomeningeal cyst, Poroencephalic cyst

\section{Introduction}

Since Howship's report of a "partial absorption of the parietal bone" [1] and the first pathological description of that condition [2] it has become known most frequently under the name, given by Pia and Tönnis [3], of Growing Skull Fracture (GSF). Skull erosion and post-traumatic leptomeningeal cyst are other appellations given to this same nosological entity that consists of a skull fracture that evolves into a skull defect.

GSF has become a well-recognized entity, although an infrequently found pathology in the pediatric head trauma population. Ninety per cent of GSF is encountered in children under the age of 3 -years $[4,5]$. Its detection in adult life but stemming from a childhood head trauma is occasionally encountered in clinical practice $[6,7]$, but its occurrence in adult life, derived from an adult head trauma is indeed rare [8].

\section{Material and Methods}

A careful search of the literature for the headings of "skull fracture" "leptomeningeal cyst" and "skull erosion" was carried out and the information obtained clarified the pathological elements necessary for the genesis of GSF. The basic laws of hydraulics were applied to that pathological substrate, to develop a clearer understanding of the GSF genesis and development.

\section{Discussion}

Two undisputed factors in the genesis of GSF are a gaping skull fracture and an associated underlying dural tear. Any simultaneous injury to the neighboring meninges and/ or brain tissue constitutes a secondary pathogenetic element. Moreover, the rapid growth of the brain and skull in infancy, which is maximal during the first year of life, has been considered still another additional pathogenetic factor [9]. Finally, by altering the direction of the dural vectors of intracranial pressure [10], the resulting skull defect may be responsible for the asymmetrical skull growth sometimes associated with GSF $[6,9]$.

GSF may occur at the skull convexity, the parietal region being its most frequent location. Rarely GSF can develop at the cranial base. While basilar skull fractures occur in about $4 \%$ of severe head injuries and represent $15-21 \%$ of all skull fractures [11], their potential complications are relatively frequent and well known, but its evolution into a growing basilar skull fracture is rare.

When the GSF is analyzed in a temporal framework, three different types emerge: 1) A CHILDHOOD type, where the initial trauma, its clinical evolution, its diagnosis and treatment all occur during that period of life. It is by far the most frequent case found in clinical practice; 2) A DELAYED type, where the initial trauma does occur during childhood but it is not diagnosed nor treated until adulthood; 3) Finally an

*Corresponding author: Allan J Drapkin, MD, FACS(R), Department of Neurosurgery, Rutgers Biomedical and Health Sciences University, Alejandro Serani Norte, 9458 Apt. 402 Vitacura, Santiago, Chile, USA, Tel: +562229801357

Accepted: June 12, 2021

Published online: June 14, 2021

Citation: Drapkin AJ (2021) Growing Skull Fracture [GSF], a Hydrodynamic Perspective: An Original Article. J Pediatr Neurol Neurosci 5(1):131-133 
ADULT type, where the initial trauma and its later evolution, diagnosis and treatment occur in adulthood.

When GSF is studied in its pathologic context, it can present in four different forms: 1) As SKULL EROSION, where it causes a through and through skull defect; 2 ) As an INTRADIPLOIC CYST when the GSF destroys only the inner table of the skull, expands into the diploe, thinning and deforming of the skull outer table; 3) BRAIN HERNIATION which occurs when the resulting skull defect is occupied by brain tissue herniating through the dural defect and 4) LEPTOMENINGEAL CYST. Here the skull defect is occupied by leptomeninges filled with CSF herniating through the dural defect which can occasionally be associated with a CSF fistula [12].

GSF appearances on plain X-rays to manifest itself as a roughly elliptical skull defect with everted and scalloped margins [4]. Occasionally a portion of the original linear skull fracture can still be seen connecting at some point to the skull defect. According to their CT presentation, GSF can be grouped in three types [13]: Type I) Where the GSF area is occupied by a leptomeningeal cyst; Type II) Here the GSF is occupied by herniating brain tissue and Type III) Where the GSF is filled by a Poroencephalic cyst. Similar groupings can be found when GSF is studied by MRI.

Finally, the GSF clinical presentation can manifest itself in different ways: 1) As focal or generalized seizures or as persisting headache, either localized or generalized; 2) It can also present itself as a neurological deficit such as hemiparesis or developmental delay or as a scalp mass, pulsating or not [14]; 3) Finally it can also appear as a visible and/or palpable skull defect which must be differentiated from other types of skull lesions. Regardless of the period of time elapsed since the original trauma, the dura mater tear does not heal and the dural defect expands becoming usually larger than the eroded skull defect, a fact that can be clearly seen when any GSF is submitted to surgical intervention. This inadequate dural capacity for repair has been demonstrated also in the laboratory [15] where if a dural defect is left in contact only with the skull bone, it does not heal, however, when the dural defect is placed experimentally adjacent to soft tissues (periost, galea aponeurotica or muscle) the dural defect scars and will eventually heal. Notwithstanding that these conditions are present in any GSF, no dural healing occurs, stressing the need for the recommended dural surgical repair. This quandary seems to be explained by the presence of tissue herniating through the dural defect (leptomeninges, brain tissue, or a poroencephalic cyst) which, by its physical presence in between the lips of the dural tear and its constant pulsation there, it would prevent the dural reparative process.

An analysis of published cases of GSF reveals its relatively frequent association with an underlying poroencephalic cyst and, at times also with a dilated portion of the ipsilateral ventricle which is shifted towards the skull defect [9]. It has been demonstrated experimentally that the creation of a skull defect with a dural laceration, results in an increased distensibility of the brain tissue in that area $[16,17]$. Nevertheless, this change in the elastic properties of the brain tissue becomes manifest only when an additional force comes into play, such as an increased intraventricular pressure (as in hydrocephalus) or as a decreased regional tissue pressure (as it occurs when brain edema resolves or when a localized area of injured brain is reabsorbed). This fact, and depending on the dimensions of the area of brain tissue affected, could lead to a poroencephalic cyst underlying the dural defect and/or herniating into the GSF or if the affected area is larger, due to a partial dilation of a ipsilateral ventricle which, because of the decreased tissue pressure existing in that area, would be shifted towards the skull defect.

Although the enlargement of the skull defect in a GSF may attain its final dimensions rather rapidly, particularly when it develops in infancy, it will eventually reach an arrest in growth and the skull defect may remain quiescent for varying periods of time, even for many years. In the past, this fact led to the advice given for only conservative treatment in GSF, without the need for surgical intervention [18]. Nevertheless, clinical experience has proven that GSF may produce clinical symptoms, at times severe, during the time period in which the bone defect remains stationary $[6,13]$. It becomes evident then that it is the unhealed dural defect, with the leptomeninges and/or brain tissue herniating through it that represents the vulnerable spot capable of causing this late symptomatology and that justifies and demands its surgical repair.

This surgery should include the dural repair and a cranioplasty, performed preferably with autologous bone harvested from the inner table of the skull surrounding the GSF.

While a ventriculoperitoneal shunts have been occasionally used as the sole treatment for GSF because of the commonly associated ventriculomegaly, [19] this method does not correct the dural defect and would leave the patient prone to experience the complications of GSF should the shunt malfunction.

\section{Proposed Pathogenetic Mechanism for GSF}

Herniation of the leptomeninges through the dural defect propelled by the constant pulsation of the cerebrospinal fluid (CSF) they contain [20] would bring them eventually in contact with the skull fracture edges. The CSF being a compressible fluid, follows the hydraulic principle of Pascal, according to which "it will transmit any pressure applied to it, in all directions, with undiminished force and throughout its container." Furthermore, when the fluid pulsations are suddenly brought to a stop or forced to change its direction of flow, as it would occur when the pulsating CSF hits the fracture edges, it would cause a momentum change and a pressure surge at that site (the water hammer effect). This elevated pulse pressure would cause an increased bone erosion at the fracture edges and a progressive skull destruction leading to a GSF. This progressive enlargement of the bone defect, brought about by the on-going bone erosion, will eventually result in a gradual decrease in the blocking effect exerted by the fracture edges upon the CSF flow, causing a gradual reduction in the momentum change imposed upon the CSF pulse wave in that area. This would result in a gradual decrease and eventual cessation of the pressure surge generated there and would progressively reduce the bone erosion. In due time, it would 
lead to the stabilization of the skull defect.

\section{Conclusion}

While in GSF the skull erosion will eventually cease, the dural tear does not heal, causing the eventual development of progressive symptoms and/or neurological deficits. This requires the surgical correction of any GSF with repair of that dural defect.

\section{Acknowledgment}

The author wishes to thank Paola D. Vermeer PhD and Ms. Elizabeth Gonzalez for their technical and bibliographical assistance in the performance of this work.

\section{Declarations}

\section{Conflict of interest}

The author certifies that he does not have affiliations with or involvement in any organization or entity with any financial or non-financial interest in the subject matter discussed in this manuscript.

\section{Ethics statement}

Written informed consent was obtained from the individual for the publication of any potentially identifiable data included in this article.

\section{References}

1. Howship J (1816) Practical observations in surgery and morbid anatomy. London: Longman Hurst. Rees. Orme and Brown.

2. Rokitansky CV (1856) Lehrbuch der pathologischenanatomie. (edn 3) Viena W Braumuller, 2.

3. Pia HM, TönnisW (1953) Die Wachsende Schädelfractur des kindesalters. Zentralblattfür Neurochirurgie 13: 1-23.

4. Kingsley D, Till K, Hoare R (1978) Growing fractures of the skull. Journal of Neurology, Neurosurgery, and Psychiatry 41: 312-318.

5. Lende RA, Erickson TC (1961) Growing skull fractures of childhood. J Neurosurg 32: 680-683.

6. Drapkin AJ (2006) Growing skull fracture: A posttraumatic neosuture. Childs Nerv Syst 22: 394-397.
7. Singla N, Gupta KS (2010) The natural history of an untreated growing skull fracture: An unusual case. Pediatr Neurosurg 46: 76-79.

8. Halliday AC, Chapman PH, Heros RC (1990) Leptomeningeal cyst resulting from adult trauma. Case report. Neurosurgery 26: 150153.

9. Scarfo GB, Mariottini A, Tomaccini D (1989) Growing skull fractures: Progressive evolution of brain damage and effectiveness of surgical treatment. Child's Nerv Syst 5: 163-167.

10. Moss ML (1975) Functional anatomy of cranial synostosis. Child's Brain 1: 22-54.

11. Mokolane NS, Minne C, Dehnavi A (2019) Prevalence and pattern of basal skull fracture in head injury patients in an academic hospital. SA J Radiol 23: 1677-1684.

12. Saurabh J, Ashok G, Achal S, et al. (2015) Growing skull fracture with cerebrospinal fistula: A rare case report and its management. Asian J Neurosurg 10: 228-231.

13. Harter DH, Swanger R, Tenner M (2004) Growing burr hole: Enlarging pseudo meningocele at the site of a craniotomy. Child's Nerv Syst 20: 127-130.

14. Muhonen MG, Piper JG, Menezes AH (1995) Pathogenesis and treatment of growing skull fractures. Surgical Neurology 43: 367373.

15. Keener EB (1959) An experimental study of reactions of the dura mater to wounding and loss of substance. J Neurosurg 16: 424447.

16. Drapkin AJ, Sahar A (1978) Experimental hydrocephalus: Cebrospinal fluid dynamics and Ventricular distensibility during early stages. Child's Brain 4: 278-288.

17. Hochwald GM, Epstein F, Malhan C, et al. (1972) The role of the skull and dura mater in experimental feline hydrocephalus. Dev Med Child Neurol Suppl 27: 65-69.

18. Ramamurthi B (1970) Secondary trauma in an adult skull. J Neurosurg 32: 427-430.

19. Galbraith A (1982) Letter to the Editor. J Neurosurg 56: 169.

20. Brooks ML, Jolesz FA, Patz S (1988) MRI pulsatile motion within arachnoidal cysts. Magnetic Resonance Imaging 6: 575-594.

DOI: $10.36959 / 595 / 421$

Copyright: (C) 2021 Drapkin AJ. This is an open-access article distributed under the terms of the Creative Commons Attribution License, which permits unrestricted use, distribution, and reproduction in any medium, provided the original author and source are credited. 